\section{tRNA and mRNA both in the same molecule}

Sir-The recent discovery of single protein monomers synthesized from two messenger RNAs due to ribosomes switching from one mRNA to another ${ }^{1,2}$ raises provocative mechanistic issues. The relevant feature of the first mRNA is that it lacks a stop codon ${ }^{1}$, while the second mRNA is 10Sa RNA (the suggested name is tmRNA), a small stable non-ribosomal RNA found in a wide variety of procaryotes ${ }^{3,4}$. The proposed model is that tmRNA, aminoacylated with alanine, enters the A site of a ribosome stalled at the $3^{\prime}$ end of an mRNA that lacks a terminator, and donates alanine to the nascent peptide. It then functions as mRNA with resultant freeing of trapped ribosomes (ref. 1, H. Himeno and A. Muto, personal communication). How does tmRNA fit into the ribosome to function first as a tRNA, and second as a mRNA?

Previously, pairing of the $5^{\prime}$ and $3^{\prime}$ ends of tmRNA has been proposed to result in the formation of a tRNA acceptor stem with sequences close to the $3^{\prime}$ end having the potential to form a T $\psi \mathrm{C}$-like stem-loop $^{4,5}$. On the basis of sequence comparisons from ten different microorganisms, we find that there is a strong sequence conservation in many parts of the molecules and, focusing on the $3^{\prime}$ and 5 ends, we have extended the structural analogy of tmRNAs with tRNAs to one of full mimicry. A variable loop, an anticodon stemloop and a D stem-loop are proposed (Fig. 1), so that the overall shape of the ends of the molecules should allow them to fit into the ribosomal A-site. In these tRNA analogues, the proposed anticodon-
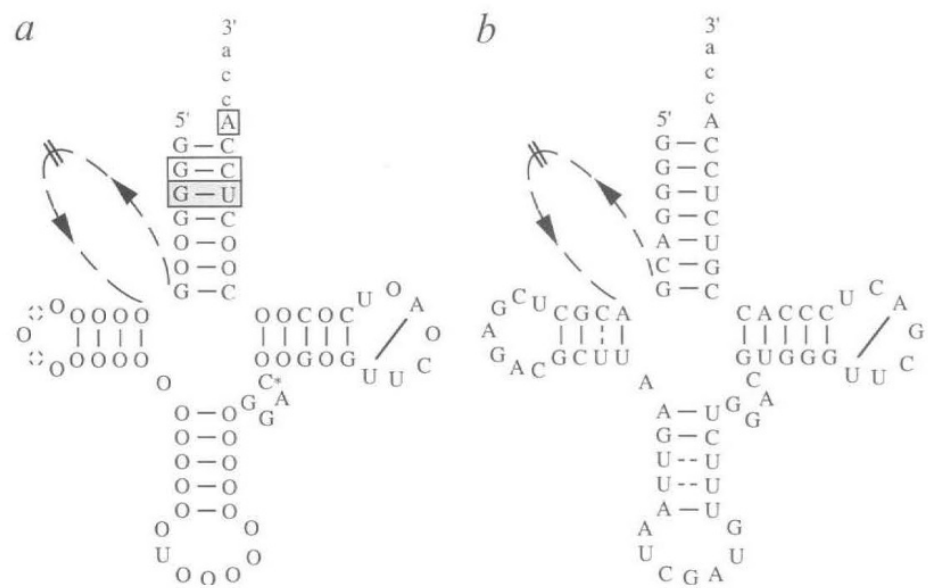

Fig. 1 Analogy of tmRNAs to canonical tRNAs. $a$, The proposed consensus cloverleaf structure for tmRNAs from Escherichia coli. Haemophilus influenzae, Mycobacterium tuberculosis, Bacillus subtilis, Mycoplasma capricolum and $M$. genitalium, Alcaligenes eutrophus, Dichelobacter nodosus and Vibrio cholerae. Strictly conserved nucleotides are in capital letters, and variable nucleotides are shown as circles. Nucleotides in the shaded box are identity elements for aminoacylation with alanine, and nucleotides that are simply boxed are minor identity elements ${ }^{6}$. Bars represent proposed base-pairs, lower case letters indicate nucleotides that can be added post-transcriptionally. * signifies that one species possesses an adenine residue, and dashed lines correspond to about 290 nucleotides. b. Proposed folding of Bacillus subtilis tmRNA. In E. coli, H. influenzae and $V$. cholerae, the apparent acceptor stem has four canonical base pairs. Note that a conserved $\mathrm{A}-U$ base pair in the T $\psi \mathrm{C}$-like loop mimics the reversed Hoogsteen interaction found in canonical tRNAs. For all these molecules, the variable loop has been changed from three to five nucleotides in length, and the better foldings were observed for a four-nucleotides loop. However, we could not exclude that its size may vary according to the species.

like branch varies in sequence, but its secondary structure can be maintained. [In most cases a conserved uridine is located at a position analogous to that of the $\mathrm{U}_{33}$ residue in canonical tRNAs, needed for a correct folding of the anticodon loop (U-turn)]. With geometry resembling that of canonical tRNAs, tmRNAs may fill the decoding site on the small subunit. Variation of the anticodon sequences suggests that their identities are unimportant-perhaps not surprising since codon-anticodon pairing presumably does not occur. The predicted 'variable loop' sequence, 5'-GGAC-3', is conserved in tmRNAs, and could interact with some ribosomal components to facilitate the folding needed for its subsequent function as mRNA. However, there is a conserved sequence on tmRNAs 65 nucleotides $5^{\prime}$ of the variable loop which could pair exactly through a tertiary interaction (in one case, covariation is seen). We conclude that a full tRNA-like structure likely forms for subsequent aminoacylation and recognition by the ribosome. How the tmRNA performs its second function, as mRNA, is hard to envision-perhaps there is a different structural conformation in which the coding domain is fully accessible for translation. Probing the structure of tmRNA, should be informative.

Brice Felden', John F. Atkins², Raymond F. Gesteland ${ }^{1,2}$

${ }^{1}$ Howard Hughes Medical institute and 2Department of Human Genetics, 6160 Eccles Genetics Building, University of Utah, Salt Lake City, Utah 84112, USA

Correspondence should be addressed to J.F.A.

Received 11 March, accepted 17 April 1996.

1. Keiler, K.C., Waller, P.R.H. \& Sauer, R.T. Science 271, 990-993 (1996).

2. Tu, G.F., Reid, G.E., Zhang, J-G., Moritz, R.L. \& Simpson, R.J. J. Biol. Chem. 270, 9322-9326 (1995).

3. Chauhan, A.K. \& Apirion, D. Mol. Microb. 3 , 1481-1485 (1989)

4. Ushida, C. Himeno, H. \& Watanabe, T. \& Muto, A. Nucl. Acids Res. 22, 3392-3396 (1994)

5. Komine, Y., Kitabatake, M., Yokogawa, T., Nishikawa, K. \& Inokuchi, H. Proc. Natl. Acad. Sci. USA 91, 9223-9227 (1994).

6. Francklyn, C. \& Schimmel, P. Nature 337. $478-481$ (1989). 